\title{
LC3-I conversion to LC3-II does not necessarily result in complete autophagy
}

\author{
POL GIMÉNEZ-XAVIER ${ }^{1}$, ROSER FRANCISCO ${ }^{1}$, FRANCESCA PLATINI ${ }^{2}$, \\ RICARDO PÉREZ ${ }^{2}$ and SANTIAGO AMBROSIO ${ }^{1}$ \\ ${ }^{1}$ Unitat de Bioquímica, Departament de Ciències Fisiològiques II; \\ ${ }^{2}$ Unitat de Biologia Cellular, Departament de Patologia Experimental i Terapèutica, \\ Campus de Ciències de la Salut, IDIBELL, Universitat de Barcelona, Barcelona, Spain
}

Received June 17, 2008; Accepted August 8, 2008

DOI: 10.3892/ijmm_00000085

\begin{abstract}
Autophagy was induced in human neuroblastoma SH-SY5Y cells by two different procedures: deprivation of fetal serum in culture medium, or treatment with dopamine. 3-methyladenine prevented autophagy in the two procedures. Although it is usually considered that the conversion of soluble LC3-I to lipid bound LC3-II is associated with the formation of autophagosomes, the inhibition of autophagy with 3-methyladenine prevented this transformation in serum-deprived but not in dopamine-treated cells. While the PI3K-mTOR pathway was inhibited by serum deprivation, dopamine increased the phosphorylation of Akt but inhibited mTOR activity in a similar way to rapamycin. Dopamine and rapamycin increased LC3-II levels by a mechanism not prevented by 3-methyladenine. The activation of LC3-I to LC3-II may then be necessary but not sufficient to trigger cell autophagy. Thus, the increase in LC3-II, as the main biochemical parameter for autophagy at present, should be considered with caution.
\end{abstract}

\section{Introduction}

Autophagy is a process by which the cell digests components or organelles of its own cytoplasm as a response to lack of nourishment, the effect of certain cytotoxins, the presence of non-functional protein aggregates (aggresomes) or in order to remove damaged organelles $(1,2)$.

The research on autophagy as a mechanism for regulating cell death or survival has acquired huge interest in recent years, not only as a cell response to starvation but also as a mechanism involved in tumorigenesis, neurodegeneration

Correspondence to: Dr Santiago Ambrosio, Unitat de Bioquímica, Departament de Ciències Fisiològiques II, Campus de Bellvitge, Universitat de Barcelona, c/Feixa Llarga s/n. Hospitalet del Llobregat, 08907 Barcelona, Spain

E-mail:sambrosio@ub.edu.

Key words: autophagy, LC3, 3-methyladenine, SH-SY5Y cells and non-caspase-linked forms of cell death (3). The molecular mechanisms of autophagy are being progressively elucidated from proteins initially described in yeast (4), Apg or Aut proteins. The mammalian homolog proteins received the name Atg proteins (1) and several of them were found to be previously known but without well-defined functions. One of these is the Atg 8 protein, known also as microtubuleassociated protein 1 (MAP1) light chain 3 (MAP-LC3 or simply LC3). These proteins covalently bind phospholipids and are components of the autophagic vacuole membranes (5). They exist as a soluble free form, LC3-I, and a LC3-II lipoprotein, covalently linked to a phosphatidylethanolamine, with a higher electrophoretic mobility in SDS-PAGE due to their increased hydrophobicity (6). The LC3-I free form is converted to the LC3-II form after being cleaved by a Cys-protease known as autophagin or Atg-4, activated by reactive oxygen species produced during starvation, or by other conditions leading to autophagy (7). This step is necessary for the action of an E1 enzyme, Atg-7, which triggers two conjugation machineries: one leading to Atg-5/ Atg-12 conjugation and subsequent linkage to Atg16, and another leading to the conjugation of LC3 to phospholipids (LC3-II), the two are required to generate a cytosolic autophagic vacuole de novo $(1,2)$. The two processes are interdependent but their mechanistic relationship is, as yet, unknown. LC3-II remains linked to the membrane of the autophagolysosome, generated after the fusion of the autophagic vacuole with the lysosome. Western blot assessment of the increase in either the LC3-II form or of the relationship between LC3-II and LC3-I content is currently considered as a simple, quick procedure to verify the presence of cell autophagy (2) and its use is advised in recently published guidelines for the interpretation of assays monitoring autophagy (8).

Various procedures have been applied to study the consequences of blocking autophagy: the use of siRNAs of Atg proteins, the inhibition of lysosome-autophagosome fusion with the microbial toxin bafilomycin and, the simplest and most useful procedure, the use of 3-methyladenine (3-MA). This compound inhibits autophagy completely (9), leading the cell either to survival or to apoptosis according to different reports using different cell models (10). There is agreement that complete inhibition of autophagy is achieved only with 
3-MA concentrations near $10 \mathrm{mM}$ (9). These very high concentrations may cause a range of effects which have only been described in part (11). The parameters generally accepted as indicative of autophagy are the presence of autophagic vacuoles and the activation of LC3-I to LC3-II (6), the two of them supposedly blocked by 3-MA. Here, however, we report an apparent paradox: in some cases, though it prevents autophagy, 3-MA maintains LC3 in its LC3-II form. The significance of these results is discussed in connection with the interpretation of the action of 3-MA and the mechanisms of autophagy.

\section{Materials and methods}

Cell culture. SH-SY5Y human neuroblastoma cells were used (American Type Culture Collection), grown in Dulbecco's Modified Eagle Medium (DMEM, Gibco BRL) supplemented with $10 \%$ fetal bovine serum (FBS, Gibco BRL), $100 \mathrm{U} / \mathrm{ml}$ penicillin and $100 \mu \mathrm{g} / \mathrm{ml}$ streptomycin, $10 \% \mathrm{CO}_{2}$ at $37^{\circ} \mathrm{C}$. Medium without fetal serum was used to induce autophagy. Cells normally cultured with $10 \%$ FBS were treated with $100 \mu \mathrm{M}$ or $500 \mu \mathrm{M}$ dopamine (DA, Sigma) for $12 \mathrm{~h}$. Experiments were performed at $\sim 80 \%$ cell confluence. 3 Methyladenine was from Sigma-Aldrich, rapamycin and LY294002 from Calbiochem.

Microscopy. Autophagic vacuoles were detected under fluorescence microscopy with monodansylcadaverine (MDC, Sigma, $50 \mu \mathrm{M}$ for $60 \mathrm{~min}$ at $37^{\circ} \mathrm{C}$ ), as described by Larsen et al (13), and by electron microscopy (fixed in $2.5 \%$ glutaraldehyde and postfixed in $2 \%$ osmium tetroxide for $2 \mathrm{~h}$, dehydrated in ethanol and propylenoxide and embedded in araldite). In the last case, ultrathin sections were stained with uranyl acetate and lead citrate and observed by electron microscopy (Hitachi 600). For immunohistochemical LC3 fluorescent staining, fixed cells were incubated with primary antibody (anti-LC3 Santa Cruz Biotech. 1:200) and with rabbit Alexa 488-green (Molecular Probes) antibody (1:400).

Western blot analysis (WB). Equal amounts of protein (determined by the BCA reagent, Pierce) were loaded on to each electrophoresis lane on SDS-polyacrylamide gels with Tris-glycine running buffer. Nitrocellulose membranes with transfected proteins were incubated with anti LC3 antibodies (a kind gift from Dr T. Yoshimori, N.I.G. Shizuoka-ken, Japan), Beclin-1 (BD Transd Lab), Atg-5L (Abgent), Atg-12L (Abgent), phospho-Akt (Ser473, Cell Signaling), phosphoAkt (Thr308, Cell Signaling), phopsho-p70S6Kinase (Thr389, Cell Signaling), and $\alpha$-tubulin (Sigma). A biotinylated secondary antibody labeled with horseradish peroxidase (Amersham, diluted 1:1000) was used and the protein bands were observed with the chemiluminescence ECL Western blotting system (Hyperfilm ECL, Amersham).

\section{Results}

Autophagy was induced in SH-SY5Y neuroblastoma cells by $12 \mathrm{~h}$ fetal serum deprivation or by $6-12 \mathrm{~h}$ of treatment with $500 \mu \mathrm{M}$ dopamine (14) and assessed with MDC. Cells were treated with $10 \mathrm{mM} 3-\mathrm{MA}$ for $1 \mathrm{~h}$ before the medium was replaced with a serum-deprived one (S.D.) or one with $10 \%$ FBS+DA, containing the same concentration of 3-MA. 3-MA inhibited the autophagic morphology in all the conditions studied. Fig. 1 shows the effects of 3-MA at $12 \mathrm{~h}$ : the MDC granulation, characteristic of autophagy, disappeared after 3-MA treatment, in serum-deprived and dopamine-treated cells (Fig. 1A). We confirmed our previous results (14) by electron microscopy, demonstrating that $500 \mu \mathrm{M}$ dopamine treatment for $12 \mathrm{~h}$, induced cell autophagy and that 3-MA treatment prevented it (Fig. 1B).

LC3 was analyzed by WB (Fig. 2A,B) and by immunocytochemistry (Fig. 2C). LC3-II presented a clear increase in DA-treated and serum-deprived cells. The ratio between LC3-II and LC3-I was significantly increased in S.D. and DA treatment. However, on the advice of Mizishuma and Yoshimori (6), we presented the densitometry of the LC3-II band. 3-MA blocked the LC3-II increase in S.D. cells, but not in DA-treated cells. Immunocytochemistry demonstrated that LC3 was associated only with a granulose morphology related to autophagy that decreased or disappeared with 3-MA, although a strong diffuse green fluorescence persisted in DA+3-MA-treated cells, suggesting that LC-3 was increased but that its linkage to vesicular structures was not (Fig. 2C). Similar results were obtained when cells were treated with $10 \mathrm{nM}$ rapamycin, an inhibitor of mTOR: LC3-II increased and 3-MA did not prevent that increase (Fig. 3A).

Beclin (Atg-6), Atg-5 and Atg-12 were assayed by WB but no differences were found in their content after either S.D. or DA treatment (results not shown).

As the PI3K-mTOR pathway is the most described mechanism for preventing autophagy and since mTORp70S6K inhibition is often used as a procedure to trigger it $(15,16)$, we analyzed the effects of S.D. and DA with or without 3-MA on Akt and S6K phosphorylation. Since Thr-308 and Ser-473 phosphorylations are required for Akt activation (16), selective antibodies were used to distinguish between the two phosphorylations by WB. While S.D. decreased phosphorylation on Ser, $500 \mu \mathrm{M}$ DA treatment greatly increased Ser and Thr phosphorylation. 3-MA did not clearly modify phospho-Thr, but it dramatically reduced phosphoSer in S.D. and phospho-Ser and phospho-Thr in DAtreatment. The involvement of PI3K in Thr-308 phosphorylation was assessed by the PI3K inhibitor LY294002 (Fig. 4B). These results suggest a basal constitutive phosphorylation in Thr that is not altered by 3-MA, and an inhibition by 3-MA of PI3K-PDK1 and PDK2 pathways. S6K phosphorylation, indicative of a good nutritional state, protein synthesis and cell proliferation, was very high in controls but almost annihilated after S.D. or $500 \mu \mathrm{M}$ DA treatment, and was sharply reduced with 3-MA alone (Fig. 4A) and also with rapamycin treatment (Fig. 3A).

\section{Discussion}

LC3-II is, at present, the leading biochemical marker for autophagy (8). Here we report that LC3-II may be increased even in certain conditions in which autophagy has been blocked by 3-MA. This strong inhibitor of autophagy prevented the formation of autophagic vacuoles in human neuroblastoma under serum deprivation and after treatment 
A)

C
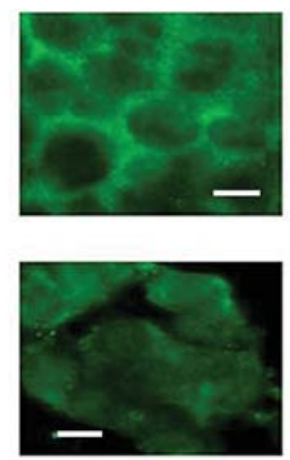

3MA
SD
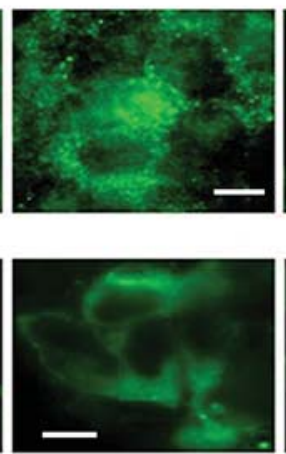

SD+3MA
SD+DA
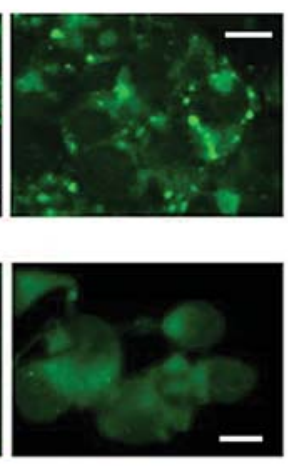

DA+3MA

B)

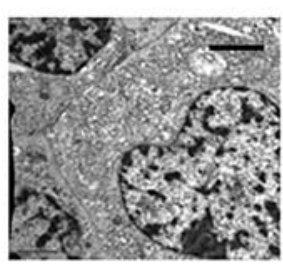

C

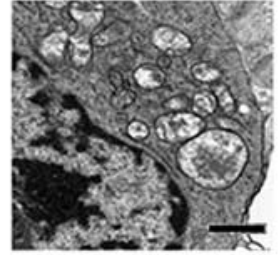

DA

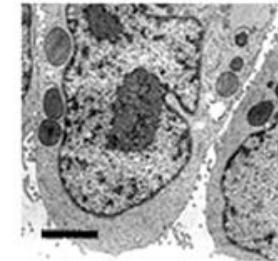

DA+3MA

Figure 1. (A) Accumulation of autophagic vacuoles in SH-SY5Y cells: controls (C), $12 \mathrm{~h}$ of serum deprivation (SD) and $12 \mathrm{~h}$ of $500 \mu \mathrm{M}$ dopamine treatment. The effect of 3-methyladenine (3MA) is shown in lower panels. Scale bar $10 \mu \mathrm{m}$. (B) Electron micrograph showing a normal control SH-SY5Y cell (C) and cells after $12 \mathrm{~h}$ of treatment with $500 \mu \mathrm{M}$ dopamine (DA) or dopamine plus $10 \mathrm{mM}$ 3-MA (DA+3MA). Scale bar $1 \mu \mathrm{m}$.

with dopamine. The specific targets of 3-MA, at the concentrations needed to block autophagy, are not well defined. 3-MA has been described as a low affinity inhibitor of PI3K activities (17), mainly PI3K class-III (PI3K-III), not linked to growth factor receptors but to intracellular membranes of the trans-Golgi network, the mammalian homolog of the yeast form Vps34 $(12,18)$.

The molecular mechanisms involved in autophagy are being investigated $(1,2)$. However, many aspects are still difficult to understand, and certain mechanistic differences probably underlie the different causes of the process. PI3K activity appears to be a key factor in triggering autophagy and PI3K inhibitors (wortmannin and LY294002) are often used (with varying degrees of success) to prevent it (19). PI3K class-III forms an active complex with Beclin-1, negatively regulated by Bcl-2 or Bcl-x (20). PtdIns-3-P, generated by PI3K-III, triggers the Atg machinery responsible for autophagy (1). PtdIns-3,4,5-P3, generated by PI3K class-I linked to growth factor receptors (PI3K-I), targets Akt/protein kinase $\mathrm{B}$ (PKB) on Thr-308 through PDK-1 activity. Akt must be further phosphorylated in Ser-473 by PDK-2 activity. Among other processes, active Akt regulates the mTOR pathway, enhancing protein synthesis and cell survival (16). However, the mTOR pathway is clearly involved in preventing autophagy, and therefore the effect it exerts is the opposite to that of PI3K. Whereas the mTOR-Raptor complex (sensitive to rapamycin) activates the phosphorylation of p70S6K, and thus protein synthesis, the mTOR-Rictor complex (insensitive to rapamycin) is probably responsible for PDK-2 activity (16). mTOR-Raptor must negatively control autophagic signals acting through growth factors or PI3K-III, so LC3 is likely to be maintained in its soluble LC3-I form providing that the mTOR pathway is active.

In a cell culture without fetal serum, the mTOR pathway is practically inactive due to the absence of growth factor stimulation, and therefore S6K is not phosphorylated. PI3K-III may then trigger LC3 activation and autophagy. In this case, 3-MA or specific PI3K inhibitors prevent the autophagy morphology and the conversion of LC3-I to LC3-II (19).

When autophagy was induced with a chemical agent such as dopamine (14), 3-MA blocked autophagy but did not inhibit the LC3-II increase. LC3 is thus linked to phospholipids and probably binds intracellular membranes [preautophagosome structures (21)], but these structures did not develop into autophagic vacuoles. The toxic action of DA in neuroblastoma (and probably in neurons) is a complex process, involving oxygen radical production, cell redox imbalance, mitochondrial function impairment, and changes in expression and activity of a number of proteins with a role in cell survival or death $(14,22)$. After DA treatment, Aktphosphorylated forms greatly increased (presumably by reducing the turnover of this protein; study of this issue is 

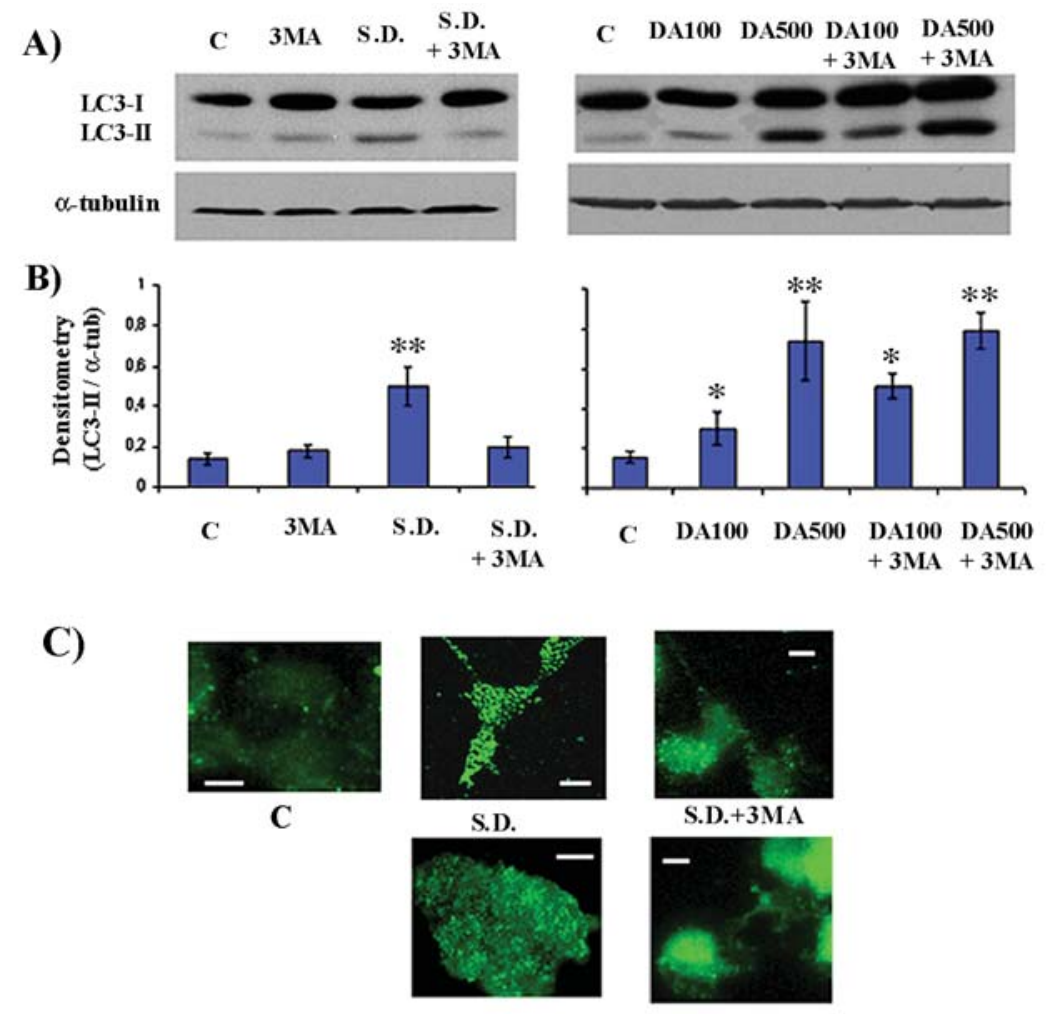

DA500

DA500+3MA

Figure 2. Western blot of LC3 bands (A) and densitometry of LC3-II band related to $\alpha$-tubulin (B), $12 \mathrm{~h}$ after serum deprivation (S.D.) or 100-500 $\mu \mathrm{M}$ dopamine treatment (DA100 and DA500), with or without $10 \mathrm{mM}$ 3-MA. Results are expressed as the mean of relationships between densitometries of LC3-II and $\alpha$-tubulin bands \pm SEM for three different experiments. ANOVA plus Scheffe test: $P \leq 0.05 ;{ }^{* *} \mathrm{P} \leq 0.01$. (C) LC3 distribution by immunofluorescence $12 \mathrm{~h}$ after serum deprivation (S.D.) or $500 \mu \mathrm{M}$ dopamine treatment (DA500). 3-MA clearly reduced LC3 staining and granulose feature in S.D. cells. In dopaminetreated cells the fluorescence intensity was high with or without 3-MA, but in the latter condition the granulose feature was lost. Scale bar $10 \mu \mathrm{m}$.

A)

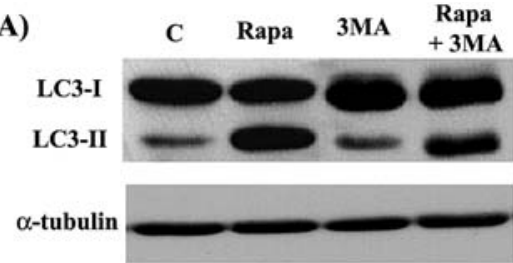

B)

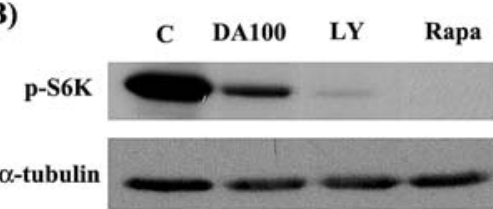

Figure 3. Western blot of LC3 after treatment with $10 \mathrm{nM}$ rapamycin (Rapa) and $10 \mathrm{mM}$ 3-MA (A), and 70S6K(phosphoThr389) (p-S6K) after treatment with $100 \mu \mathrm{M}$ dopamine (DA100), $20 \mu \mathrm{M}$ LY294002 (LY) or $10 \mathrm{nM}$ rapamycin (Rapa) (B). All the treatments were carried out for $12 \mathrm{~h}$. The image is representative of three different experiments.

currently underway in our laboratory), but, downstream of Akt, the mTOR-Raptor pathway was inhibited and S6K inactivated. LC3-I was then converted to LC3-II and autophagy took place as a mechanism to counteract the hypoxic-like condition caused by DA. A similar effect was caused by the mTOR inhibitor rapamycin. In these cases 3-MA was unable to prevent LC3 activation, although autophagy was inhibited.

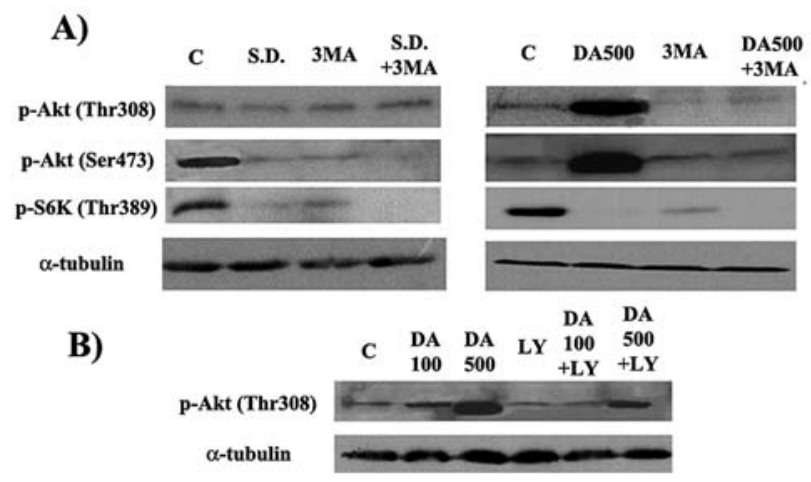

Figure 4. Western blot of Akt(phosphoThr308), Akt(phosphoSer473) and p70S6K(phosphoThr389) after $12 \mathrm{~h}$ of serum deprivation or $500 \mu \mathrm{M}$ dopamine treatment, with or without $10 \mathrm{mM} 3-\mathrm{MA}$ (A). Western blot of Akt(phosphoThr308) after $100 \mu \mathrm{M}$ (DA100) or $500 \mu \mathrm{M}$ (DA500) dopamine treatment and co-treatment with $20 \mu \mathrm{M} \mathrm{LY} 294002$ (LY, a PI3K inhibitor), showing a reduction in both DA100 and DA500. The image is representative of three different experiments.

Increases in Akt phosphorylation on Thr-308 and Ser-473 were blocked by $3-\mathrm{MA}$, which thus seems to interfere in PDK-1 and PDK-2 pathways (16). PI3K-I-mTOR and PI3KIII pathways may be considered to have opposite effects on autophagy; however it is well accepted that the inhibition of these activities results in autophagy inhibition (12). 
These results suggest that LC3-I conversion to LC3-II is a necessary but not sufficient condition for autophagy. The effect of 3-MA has often been assayed in GFP-LC3 transfected cells, where it prevents the LC3 aggregation caused by autophagy (10), but this does not rule out the possibility that LC3-I may be converted to LC3-II without aggregation in vacuoles. It must be considered that complete autophagy needs the concomitant activation of other factors of the Atg family. Autophagy is, at present, known to participate in the pathogenesis of multiple human diseases $(23,24)$. It should be considered that, in some cases, when the aggregation leading to the autophagy inhibits the mTOR pathway, LC3-II may be increased in cells that do not develop complete autophagy.

\section{Acknowledgements}

We are grateful to all the members of the Biochemistry Units (Campus Bellvitge) of the University of Barcelona (UB) and S.A.L. at the UB for linguistic help. We thank Dr Tamyoshi Yoshimori of the Department of Cell Genetics National Institute of Genetics, Yata 1111 Mishima, Shizuokaken, Japan, for kindly providing the LC3 antibody that allowed us to carry out this study. P. Giménez-Xavier is the recipient of an FPI grant and this work was supported by grants from Fundació La Caixa (BM06-125-2) and FISS (PI06126).

\section{References}

1. Klionsky DJ: Autophagy: from phenomenology to molecular understanding in less than a decade. Nat Rev Mol Cell Biol 8: 931-937, 2007.

2. Mizushima N: Autophagy: process and function. Genes Dev 21: 2861-2873, 2007.

3. Shintani T and Klionsky DJ: Autophagy in health and disease: a double-edged sword. Science 306: 990-995, 2004.

4. Ohsumi Y: Molecular dissection of autophagy: two ubiquitinlike systems. Nat Rev Mol Cell Biol 2: 211-216, 2001.

5. Tanida I, Ueno T and Kominami E: LC3 conjugation system in mammalian autophagy. Int J Biochem Cell Biol 36: 2503-2518, 2004.

6. Mizushima N and Yoshimori T: How to interpret LC3 immunoblotting. Autophagy 3: 542-545, 2007.

7. Scherz-Shouval R, Shvets E, Fass E, Shorer H, Gil L and Elazar Z: Reactive oxygen species are essential for autophagy and specifically regulate the activity of Atg4. EMBO J 26: 1749-1760, 2007.

8. Klionsky DJ, Abeliovich H, Agostinis P, et al: Guidelines for the use and interpretation of assays for monitoring autophagy in higher eukaryotes. Autophagy 4: 1-24, 2008.
9. Seglen PO and Gordon PB: 3-Methyladenine: specific inhibitor of autophagic/lysosomal protein degradation in isolated rat hepatocytes. Proc Natl Acad Sci USA 79: 1889-1892, 1982.

10. Boya P, González-Polo RA, Casares N, et al: Inhibition of macroautophagy triggers apoptosis. Mol Cell Biol 25: 1025-1040, 2005.

11. Caro LHP, Plomp PJAM, Wolvetang EJ, Kerkhof C and Meijer AJ: 3-Methyladenine, an inhibitor of autophagy, has multiple effects on metabolism. Eur J Biochem 175: 325-329, 1988.

12. Kondo Y, Kanzawa T, Sawaya R and Kondo S: The role of autophagy in cancer development and response to therapy. Nat Rev Cancer 5: 726-734, 2005.

13. Larsen KE, Fon EA, Hastings TG, Edwards RH and Sulzer D: Methamphetamine-induced degeneration of dopaminergic neurons involves autophagy and upregulation of dopamine synthesis. J Neurosci 22: 8951-8960, 2002.

14. Gómez-Santos C, Ferrer I, Santidrián AF, Barrachina M, Gil J and Ambrosio S: Dopamine induces autophagic cell death and alpha-synuclein increase in human neuroblastoma SH-SY5Y cells. J Neurosci Res 73: 341-350, 2003.

15. Castellino RC and Durden DL: Mechanisms of disease: the PI3K-Akt-PTEN signaling node - an intercept point for the control of angiogenesis in brain tumors. Nat Clin Pract Neurol 3: 682-693, 2007 .

16. Hay N: The Akt-mTOR tango and its relevance to cancer. Cancer Cell 8: 179-183, 2005.

17. Ito S, Koshikawa N, Mochizuki S and Takenaga K: 3Methyladenine suppresses cell migration and invasion of HT1080 fibrosarcoma cells through inhibiting phosphoinositide 3-kinases independently of autophagy inhibition. Int J Oncol 31: 261-268, 2007.

18. Backer JM: The regulation and function of Class III PI3Ks: novel roles for Vps34. Biochem J 410: 1-17, 2008.

19. Blommaart EF, Krause U, Schellens JP, Vreeling-Sindelarova H and Meijer AJ: The phosphatidylinositol 3-kinase inhibitors wortmannin and LY294002 inhibit autophagy in isolated rat hepatocytes. Eur J Biochem 243: 240-246, 1997.

20. Feng W, Huang S, Wu H and Zhang M: Molecular basis of Bcl-XL's target recognition versatility revealed by the structure of Bcl-xL in complex with the BH3 domain of Beclin-1. J Mol Biol 372: 223-235, 2007.

21. Xie Z and Klionsky DJ: Autophagosome formation: core machinery and adaptations. Nat Cell Biol 9: 1102-1109, 2007.

22. Giménez-Xavier P, Gómez-Santos C, Castaño E, Francisco R, Boada J, Unzeta M, Sanz E and Ambrosio S: The decrease of $\mathrm{NAD}(\mathrm{P}) \mathrm{H}$ has a prominent role in dopamine toxicity. Biochim Biophys Acta 1762: 564-574, 2006.

23. Levine B and Kroemer G: Autophagy in the pathogenesis of disease. Cell 132: 27-42, 2008

24. Martínez-Vicente $M$ and Cuervo AM: Autophagy and neurodegeneration: when the cleaning crew goes on strike. Lancet Neurol 6: 352-361, 2007. 